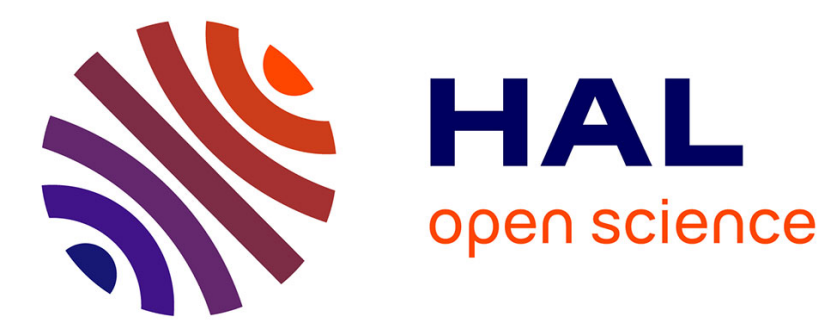

\title{
Capteurs de quotas. La pêche européenne entre territoires et marché
}

Christian Lequesne

\section{To cite this version:}

Christian Lequesne. Capteurs de quotas. La pêche européenne entre territoires et marché. Critique Internationale, 1999, 2, pp.121-131. 10.3406/criti.1999.1548 . hal-01010791

\section{HAL Id: hal-01010791 \\ https: / hal-sciencespo.archives-ouvertes.fr/hal-01010791}

Submitted on 20 Jun 2014

HAL is a multi-disciplinary open access archive for the deposit and dissemination of scientific research documents, whether they are published or not. The documents may come from teaching and research institutions in France or abroad, or from public or private research centers.
L'archive ouverte pluridisciplinaire HAL, est destinée au dépôt et à la diffusion de documents scientifiques de niveau recherche, publiés ou non, émanant des établissements d'enseignement et de recherche français ou étrangers, des laboratoires publics ou privés.

\section{(이)(\$)}

Distributed under a Creative Commons Attribution - NonCommercial - NoDerivatives| 4.0 


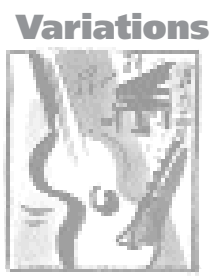

\title{
Capteurs de quotas
}

\author{
La pêche européenne \\ entre territoires et marché
}

par Christian Lequesne

ontrairement à une idée répandue, les quelque 270000 pêcheurs que compte l'Union européenne ne prennent pas la mer avec une représentation strictement individualiste de leur activité professionnelle. Ils exercent au contraire leur métier avec le sentiment d'appartenir à des communautés professionnelles ancrées dans des territoires : un État, un port, une région maritime ${ }^{1}$.

La représentation d'appartenance à une communauté professionnelle nationale trouve ses fondements dans les relations que les États ont entretenues depuis plusieurs siècles avec les « gens de mer ». Pour prendre l'exemple de la France, le régime de l'Inscription maritime, héritage d'une ordonnance de Colbert, a conféré dès le XVIIIe siècle un sens très particulier aux relations entre les pêcheurs et l'État. Son principe reposait sur une réciprocité simple : tous les « gens de mer », qu'ils fussent pêcheurs ou marins de commerce, avaient l'obligation de servir pour une période donnée dans la marine de guerre. En contrepartie, l'État leur accordait un régime spécial de pension - l'un des premiers du genre - ainsi que le monopole 
de la navigation civile et de la pêche. Si l'enrôlement obligatoire dans la marine nationale a été aboli par une loi de 1965, la contrepartie sociale de ce régime spécifique a été maintenue et adaptée aux évolutions de l'État-providence. Les « gens de mer » bénéficient toujours d'un régime dérogatoire en matière de retraites et de sécurité sociale ${ }^{2}$. Ils constituent en outre l'une des dernières professions en France sur lesquelles une administration de tutelle, les Affaires maritimes, exerce à la fois un contrôle de nature sociale (vérification des feuilles de maladie, visa des contrats de travail...) et disciplinaire (sanction des fraudes). L'époque où une administration française ouvertement paternaliste définissait son rôle à l'égard des marins-pêcheurs comme une incitation « aux institutions de prévoyance en obligeant l'épargne des hommes qui, sans elle, finiraient dans la misère, une vie usée par les fatigues d'un métier si dur $»^{3}$ a donc laissé des empreintes durables. D'autres pays européens tels que l'Espagne, l'Italie ou la Grande-Bretagne ont une histoire marquée par des relations tout aussi particulières entre l'État et les pêcheurs.

En second lieu, la majorité des marins-pêcheurs européens relient leur activité individuelle en mer avec le développement économique et social d'un territoire local, qui est le plus souvent le port d'attache de leur navire. Ce lien communautaire avec un micro-espace qui s'oppose à l'infinitude de la mer transcende les rivalités de métiers qui demeurent pourtant vives au plan local du fait des différences entre techniques de pêche, conditions de travail ou revenus. Les enquêtes que nous avons menées en France, en Espagne et en Grande-Bretagne ${ }^{4}$ montrent que la loyauté envers une communauté portuaire n'est pas seulement le fait des pêcheurs côtiers. On la retrouve chez les artisans faisant de la pêche au large, voire chez les armateurs de la pêche industrielle : lorsque Elisabeth Stevenson, partenaire de Stevenson and Sons, premier armement industriel à la pêche du Royaume-Uni, évoque les raisons pour lesquelles sa trentaine de chalutiers débarquent exclusivement leurs captures à Newlyn, elle souligne au premier chef son attachement personnel au développement du port cornouaillais dans lequel son aïeul créa la compagnie en $1889^{5}$.

\section{Pêche et politique en Europe : un compromis inter-territorial}

Ce lien communautaire entre les pêcheurs européens et des territoires transparaît dans les trois volets internes de la politique commune des pêches de l'Union européenne (PCP), créée en 1970 : l'organisation des marchés, la conservation des ressources et l'aide à la transformation des structures ${ }^{6}$; nous nous limiterons ici à la seconde, qui fait figure de noyau dur de la PCP depuis un règlement communautaire de janvier $1983^{7}$. Ce texte pose en principe de base le libre accès de tous les navires de l'Union européenne à l'ensemble des ressources halieutiques ; toutefois - et cela est tout à fait contradictoire - il réserve une bande côtière de 12 milles marins à la jouissance exclusive de chaque État riverain ${ }^{8}$. Cette dérogation 
est l'exemple même d'un compromis inter-territorial négocié par les ministres européens de la Pêche dans le seul but de protéger l'activité de leurs communautés de pêcheurs côtiers et de leurs territoires locaux respectifs9.

Un tel compromis inter-territorial se retrouve dans les mesures de conservation instituées par la PCP pour la pêche au large, c'est-à-dire dans la zone des 200 milles marins qui forme la « mer communautaire ${ }^{10}:$ la meilleure illustration en est la négociation et la fixation annuelle, par les ministres de la Pêche, des « totaux de captures autorisées » ou TAC ${ }^{11}$ s'appliquant à la capture des principales espèces et, plus encore, leur répartition en quotas nationaux. Si cette négociation prend pour point de départ des évaluations scientifiques, elle met aussi en jeu des considérations strictement politiques ayant pour unique objectif de protéger les communautés territoriales de pêcheurs de chaque État membre. À la suite du règlement de janvier 1983 et des arrangements conclus dans les Actes d'adhésion de 1985 et 1994, un principe dit de stabilité relative prévoit ainsi l'octroi annuel d'un pourcentage fixe de stocks à chaque État membre, qui n'a rien à voir avec les préoccupations des biologistes. La stabilité relative vise, d'une part, à protéger, au sein de chaque État, les besoins particuliers des régions les plus dépendantes de la pêche et les activités de pêche traditionnelle, d'autre part, à compenser les pertes de captures dans les eaux des pays tiers du fait de l'extension par ceux-ci de leurs propres zones de pêche.

Les exemples démontrant que les règles de la PCP ont été négociées avec pour principal souci un compromis inter-territorial pourraient être multipliés. Ils ne font que traduire l'attachement des pêcheurs européens à une représentation territorialisée de leur métier. Si les signes d'appartenance à des communautés

2. A. Geitsdoerfer, «L'exception maritime », Économie et Humanisme, n 335, décembre 1995, pp. 25-29.

3. Manuel Français des Pêches Maritimes, Paris, 1935, p. 98.

4. Enquêtes menées entre février 1997 et juillet 1998 auprès des principaux acteurs des pêches britannique, espagnole et française, en vue d'un ouvrage sur l'européanisation de l'action publique dans le secteur de la pêche. Les pêcheurs sont environ 70000 en Espagne, 20000 en France et autant en Grande-Bretagne.

5. Entretien, Newlyn, 6 juillet 1998.

6. Pour une histoire de la PCP, voir M. Holden (avec une remise à jour de D. Garrod), The Common Fisheries Policy, Oxford, Blackwell, 1994.

7. Règlement 170/83 instituant un régime communautaire de conservation et de gestion des ressources de pêche, fournal Officiel des Communautés européennes L 24 du 27.01.1983.

8. Unité de mesure utilisée en navigation, 1 mille marin équivaut à $1,852 \mathrm{~km}$.

9. La seule exception au régime des 12 milles est l'octroi par l'État côtier d'un droit de pêche à un nombre limité de navires d'autres États membres qui avaient une activité dans la zone avant l'établissement de la PCP. On parle alors d'un droit bistorique.

10. Reconnue par le droit international depuis la convention de Montego Bay (1982), la zone des 200 milles - appelée « zone économique exclusive » (ZEE) - est un espace qui, sans constituer une mer territoriale, permet à l'État côtier de déterminer ses propres capacités de capture ainsi que les autorisations de prises par les navires des États tiers. Dans le cadre de la PCP, la gestion des ressources halieutiques dans la zone des 200 milles est réglementée par l'UE et non par les États individuellement. Voir R. J. Dupuy, L'Océan partagé, Paris, Pédone, 1979.

11. Acronyme anglais de «total allowable catches ». 
transnationales ${ }^{12}$ fondées sur le partage d'une même espèce (comme le thon germon par les Français et les Espagnols en golfe de Gascogne) ou d'une même technique (comme le filet maillant dérivant ou le chalut de fond) ne sont pas totalement absents de leurs représentations, ils demeurent néanmoins peu marqués. Cette faible transnationalité est la principale raison pour laquelle une grande majorité des pêcheurs européens perçoit la PCP essentiellement comme une négociation diplomatique entre des États.

\section{Les surprises de la norme européenne de marché}

La logique territoriale présente des contradictions avec celle du marché, à laquelle les gouvernements européens ont accordé une priorité politique dès le traité de Rome. À compter de la fin des années quatre-vingt, le choix de réaliser, au plus tard

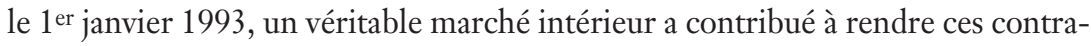
dictions plus explicites. Les pêcheurs ont alors pris conscience que les règles visant à lever les obstacles à l'édification d'un marché européen - comme la liberté d'établissement ou la libre circulation des capitaux - avaient nécessairement un impact sur leurs pratiques professionnelles fondées sur l'allégeance prioritaire à des communautés territoriales ${ }^{13}$.

Jusqu'à la fin des années soixante-dix, l'essentiel des navires pratiquant la pêche au large dans les eaux de la Communauté appartenaient ainsi à des armements dont les capitaux provenaient du seul État de pavillon. Cette situation évolua au début des années quatre-vingt lorsque des armateurs espagnols décidèrent d'exporter et d'enregistrer des navires de pêche au Royaume-Uni, afin de contourner les limites d'accès à la ressource que leur imposait l'accord bilatéral entre la Communauté européenne et leur pays. Le ministère britannique de l'Agriculture procura sans rechigner des licences à ces investisseurs étrangers attirés par l'« eldorado libéral » thatchérien. Après l'adhésion de l'Espagne le $1^{\text {er }}$ janvier 1986, un phénomène nouveau se fit jour : l'Acte d'adhésion continuant à limiter strictement l'accès des navires espagnols à la «mer communautaire » pendant une période transitoire de seize ans, des armateurs espagnols commencèrent à acheter des navires et des licences de pêche au Royaume-Uni, en particulier en Cornouailles, et à les exploiter sous pavillon britannique, comme les y autorisait le traité de Rome ${ }^{14}$.

Dans la mesure où la propriété d'un navire de pêche implique le droit d'utiliser les quotas attribués par la PCP à l'État de pavillon (compromis inter-territorial), les réactions d'hostilité des organisations de pêcheurs britanniques ne se firent pas attendre. C'est au sein de la National Federation of Fishermen's Organisations ${ }^{15}$ qu'émergea l'expression quota bopping, terme lourd de sens puisqu'il évoque le rapt par des « étrangers » des stocks de poissons appartenant aux communautés de pêcheurs britanniques. L'opposition à la « captation de quotas » 
ne fit que croître lorsqu'on s'aperçut que les armateurs espagnols ne débarquaient aucun poisson au Royaume-Uni et que des armateurs néerlandais se mettaient à leur tour à racheter des navires sous pavillon britannique. Au cours des années quatrevingt-dix, la crainte gagna les organisations professionnelles françaises qui se trouvaient désormais confrontées au même phénomène.

Face à la mobilisation des organisations nationales d'armateurs et de pêcheurs qui n'hésitaient plus à recourir à la symbolique guerrière de l'« invasion »16, les ministres des Pêches britannique et français commencèrent à inscrire la captation de quotas à l'agenda politique de leur gouvernement et de l'Union européenne. La politisation du problème visa immédiatement l'Espagne beaucoup plus que les Pays-Bas : le dynamisme d'investisseurs originaires d'un pays d'Europe du Sud semble avoir heurté la représentation que les élites politico-administratives françaises et britanniques avaient du leadership économique en Europe. Pourtant, le phénomène a toujours été quantitativement assez faible. En 1998, les navires dits « franco-espagnols » étaient au nombre de 57 (sur 6496 ) et les « anglo-spanish » environ 80 (sur 8 482). Les premiers représentaient en 1997 à peine 2,5\% de la puissance motrice totale de la flotte de pêche française. Cette part est modeste, même si elle correspond grosso modo à l'effort de réduction demandé aux professionnels français par la Commission européenne dans sa politique de réduction des flottes - d'où un sentiment d'injustice chez ces derniers ${ }^{17}$. Quant à la ponction exercée sur les quotas, principal sujet de mécontentement, la difficulté à obtenir des statistiques officielles des administrations autorise à penser qu'elle n'est peut-être pas aussi forte que les professionnels l'imaginent.

Étudiée trop souvent en fonction de ses seuls effets sur le fonctionnement de la PCP, la captation de quotas mérite d'être examinée également sous l'angle de ses origines économiques et institutionnelles. Trois éléments peuvent alors être pris en compte : la structure différenciée des marchés nationaux des produits de la pêche ; les traductions nationales différenciées de la PCP ; enfin, la protection que procure le droit de la Communauté européenne aux acteurs économiques.

12. P. Haas (ed.), Knowledge, Power and International Policy Coordination, numéro spécial de International Organization 46 (1), 1992.

13. Pour plus de détails, voir notre contribution au colloque ECPR-ISA, « Market vs. local communities ? Quota hopping and the EU fishery policy », Vienne, 16-19 septembre 1998.

14. En particulier l'article 52 CE (sur le droit d'établissement) et l'article 221 CE (sur la participation au capital des sociétés) qui deviendront respectivement les articles 43 et 294 du nouveau traité après l'entrée en vigueur du traité d'Amsterdam.

15. La NFFO est l'organisation des pêcheurs d'Angleterre et du Pays de Galles. Les pêcheurs écossais sont fédérés au sein d'une autre organisation, la Scottish Fishermen's Federation.

16. Voir par exemple Alain Parès, Affirmer la place des pêches maritimes françaises face aux défis mondiaux, rapport au Conseil économique et social, Paris, septembre 1997, p. 202.

17. Cette politique européenne de réduction des flottes correspond aux Programmes d'orientation pluriannuels (POP). Le programme actuel (POP-IV) couvre la période 1997-2002. 
Si les produits de la pêche circulent de plus en plus librement entre les pays de l'UE (en particulier depuis l'entrée en vigueur, en 1993, des règles sur le marché intérieur $)^{18}$, les marchés n'en continuent pas moins à être spécialisés par territoire et à procurer des revenus différents aux pêcheurs nationaux. Comparés à la moyenne européenne, les marchés espagnol et néerlandais offrent aux flottes nationales de bonnes capacités d'absorption de leurs captures. En raison d'un taux de consommation élevé - $39 \mathrm{~kg}$ de poissons et de crustacés par habitant et par an ${ }^{19}$ l'Espagne possède la plus grande demande intérieure en volume de l'Union européenne. Celle-ci se traduit par des prix élevés aux professionnels espagnols spécialisés dans la pêche de poissons frais de consommation courante, comme le merlu ou l'anchois, dans les eaux communautaires. Au contraire, les Pays-Bas ont un marché national prioritairement orienté vers l'exportation de produits congelés. Celui-ci procure des revenus confortables à une flotte de chalutiers modernes spécialisés dans la pêche et le conditionnement à bord d'espèces comme le maquereau ou le hareng.

Les rémunérations offertes par les marchés espagnol et néerlandais aux armateurs nationaux ont pour conséquence que ces derniers (y compris lorsqu'ils sont des patrons-artisans propriétaires d'un ou de deux navires à capitaux familiaux, comme en Galice) sont en possession de liquidités à réinvestir dans de nouveaux outils de travail. Dès lors que la PCP limite strictement la construction de tout nouveau navire afin de ne pas accroître la capacité des flottes, mais qu'il existe en revanche un marché du navire d'occasion en France et en Grande-Bretagne, le choix des armateurs espagnols ou néerlandais s'oriente vers la seconde option. Cette motivation d'ordre strictement micro-économique est une première explication du phénomène de la captation des quotas ; elle est, paradoxalement, peu soulignée par les analystes.

Ensuite, l'observation de l'action publique au sein de l'Union européenne montre que ses politiques sont moins mises en œuvre que traduites diversement par des acteurs nationaux et locaux dans des territoires qui demeurent les produits d'histoires spécifiques entre des sociétés, des marchés et des gouvernements ${ }^{20}$. La PCP n'échappe pas à ces différences. La captation de quotas s'en nourrit, comme l'illustre une comparaison de la réception des règles de la PCP relatives à la réduction des flottes en Espagne et au Royaume-Uni²1.

Depuis l'entrée en vigueur de son premier Programme d'orientation pluriannuel (POP-I) en 1983, la Direction des pêches de la Commission européenne encourage, à l'aide du budget communautaire, une réduction du nombre de navires de pêche dans le but de mettre les flottes davantage en adéquation avec les disponibilités de la ressource et les marchés tels qu'elle les évalue : un navire de pêche peut prétendre à une prime communautaire de désarmement s'il est vendu en dehors de l'UE ou envoyé à la casse. Le plus souvent, la seconde option n'est valable pour l'armateur, d'un point de vue économique, que si une aide nationale, 
et si possible régionale, vient compléter le subside communautaire. En Espagne, le gouvernement socialiste de Felipe Gonzalez, sous la pression des Autonomies (Pays basque, Galice, Andalousie) et des puissantes organisations professionnelles comme la Federación Nacional de Cofradias de Pescadores, a mis en place de telles aides complémentaires dès l'adhésion à l'Union européenne. L'impact sur les effectifs de la flotte dite de Gran Sol (celle qui opère dans les eaux de l'Union) a été significatif : de 300 navires en 1986, on est passé à 210 en 1997 (dont 125 en Galice). Au Royaume-Uni, en raison de la politique de restriction budgétaire conduite par les gouvernements Thatcher et Major, un tel système national d'aide au désarmement n'a pas été instauré. Les armateurs britanniques souhaitant cesser leur activité ont alors été tentés de vendre leur navire et leur licence au plus offrant sur le marché de l'occasion. Les armateurs espagnols - et plus particulièrement galiciens -, qui disposaient de leurs primes au désarmement venant souvent s'ajouter à un capital propre procuré par le marché, constituèrent les clients idéaux. Cet exemple illustre parfaitement combien un objectif général assigné à une politique communautaire - ici, réduire la capacité des flottes en vue de préserver la ressource peut être contourné par la diversité des processus nationaux de traduction et alimenter un effet inattendu comme la captation de quotas.

\section{Les États contournés par de nouveaux acteurs transnationaux}

Enfin, il convient de prendre en compte les ressources qu'un droit communautaire primant sur les droits nationaux offre aux acteurs économiques pour défendre leurs intérêts particuliers ${ }^{22}$. Face à des gouvernements qui ont cherché (y compris le très libéral gouvernement Thatcher) à réformer les lois nationales sur l'enregistrement des navires afin de soumettre leurs propriétaires à une condition de nationalité23, les capteurs de quotas ont compris que leur meilleure parade était l'invocation d'un droit européen d'essence libérale. Flanqués de leurs avocats, ils ont déposé des plaintes pour atteinte au libre exercice de leur activité professionnelle auprès de la Commission mais aussi des tribunaux nationaux, en sachant que ces

\footnotetext{
18. En France, 43 \% des importations de produits de la mer provenaient en 1995 des pays de l'Union européenne et $76 \%$ des exportations leur étaient destinées.

19. Contre $27 \mathrm{~kg}$ par habitant pour la France et $18 \mathrm{~kg}$ par habitant pour le Royaume-Uni, la moyenne au sein de l'Union européenne étant de $22 \mathrm{~kg}$ par habitant. Source : Eurostat, La Pêche européenne en chiffres, Luxembourg, 1998.

20. Voir Andy Smith, L'Europe politique au miroir du local. Les fonds structurels dans les zones rurales en France, en Espagne et au Royaume-Uni, Paris, L'Harmattan, 1996.

21. Ce n'est pas le seul exemple de traduction différenciée de la PCP. Pour plus de détails, voir notre contribution citée au colloque de Vienne.

22. Voir l'article de Renaud Dehousse dans ce numéro, pp. 133-150.

23. Il s'agit de la réforme de 1988 de la loi britannique dite Merchant Shipping Act.
} 
derniers ne manqueraient pas d'interroger la Cour de justice des Communautés européennes dans le cadre de la procédure de renvoi prévue par les traités. Dans plusieurs arrêts, dont l'arrêt Factortame du 13 mars 199124, les juges de Luxembourg ont déclaré, comme on pouvait s'y attendre, que le droit de la Communauté européenne ne pouvait tolérer que l'immatriculation d'un navire de pêche soit soumise à une condition de nationalité, de résidence ou de domicile (ils ont simplement considéré que l'État de pavillon pouvait exiger du propriétaire d'un navire les preuves d'un lien économique réel entre son entreprise et le territoire). Suite à l'arrêt Factortame, le gouvernement britannique est toujours engagé dans une bataille juridique destinée à éviter le paiement de quelque $100000 £$ de dommages et intérêts à des armateurs espagnols auxquels il avait interdit toute activité en 19891990 en vertu de sa nouvelle loi sur l'enregistrement des navires. À l'instar de nombreux acteurs économiques, les armateurs ont donc perçu tout l'intérêt d'utiliser la règle de droit européenne par la voie contentieuse en vue de contrer les restrictions imposées par les États à l'européanisation de leur activité.

L'étude de la captation de quotas permet d'observer de manière concrète les dynamiques d'un espace européen de représentation des intérêts dans lequel de nouveaux acteurs économiques transnationaux sont de plus en plus aptes à contourner les États en vue de satisfaire des besoins et des objectifs utilitaristes. Cela ne signifie pas que les États ont pour autant renoncé, dans un jeu à somme nulle, à réguler leurs économies nationales et leurs territoires ${ }^{25}$.

Alors qu'une grande majorité des armateurs et des pêcheurs européens se sentent liés par l'allégeance à des communautés territoriales, les capteurs de quotas illustrent, à l'inverse, l'émergence dans le paysage de la pêche européenne d'acteurs transnationaux dont l'activité comme les représentations sont clairement déterritorialisées. Si ceux-ci vendent en priorité leur production en Espagne (ou aux Pays-Bas), c'est essentiellement parce que les prix y sont les plus élevés. Lorsqu'ils vendent dans l'État de pavillon, ce sont également des considérations de marché qui entrent prioritairement en ligne de compte. Cette logique marchande induit d'ailleurs des appels à la concurrence entre ports, voire entre organisations de producteurs locaux, qui cherchent à attirer vers eux les productions des capteurs de quotas, comme on l'observe entre La Rochelle et Lorient à propos de certains navires franco-espagnols. Les armateurs vont à leur tour jouer sur cette concurrence pour préserver leur seul véritable objectif : des coûts de commercialisation aussi bas que possible. L'intérêt croissant que trouvent les acteurs économiques européens à « démonopoliser» l'allégeance à $u n$ État peut également être illustré par la flexibilité dont font preuve les compagnies à capitaux espagnols en matière de sécurité sociale des marins. La plupart des armements anglo-espagnols ou francoespagnols emploient en effet des équipages de nationalité espagnole et résidant en Espagne. En conformité avec le droit de la Communauté européenne, ces employés 
peuvent être inscrits soit à la caisse sociale des marins français ou britanniques, soit à celle des marins espagnols. Or les armements décident parfois de jouer sur les deux régimes nationaux en fonction de considérations purement utilitaristes. Une partie des équipages travaillant sur les navires à capitaux espagnols gérée par la compagnie Pronaval à Hendaye est ainsi affiliée à la caisse française des marins - l'Établissement national des invalides de la Marine - alors que l'autre partie l'est à l'équivalent espagnol - l'Instituto social de la Marina. Le régime français offrant des prestations sociales supérieures, la compagnie Pronaval l'a retenu pour ceux de ses marins qui résident à moins de deux heures de la frontière et qui peuvent facilement se faire soigner en France. Pour les autres, le choix s'est porté sur le régime espagnol qui offre des prestations inférieures mais qui est aussi moins coûteux en termes de cotisations ${ }^{26}$. Apparemment banale, cette marge de choix croissante dont disposent les acteurs économiques à l'égard des États et des lois nationales pour gérer leur activité ne l'est précisément pas dans le domaine de la pêche, puisqu'elle continue à heurter de front la représentation dominante du lien privilégié entre pêche et territoire.

C'est la raison pour laquelle la captation de quotas donne lieu à des mobilisations politiques et à des réponses de la part des États sous la forme de politiques nationales réactives. La forte charge symbolique dont la mer et les activités maritimes sont porteuses dans l'imaginaire collectif de nations comme la France ou la Grande-Bretagne contribue sans aucun doute à renforcer les mobilisations ${ }^{27}$. Au Royaume-Uni, ce n'est pas par hasard que les mouvements politiques nationalistes, opposés au projet global de l'Union européenne, en ont fait un aiguillon de leur « croisade contre Bruxelles » à partir des années quatre-vingt-dix ${ }^{28}$. Au programme du mouvement Transpartisan Campaign for an Independent Britain, créé en 1996, figure ainsi la nécessité pour une « nation de pêcheurs » de « sauver le poisson britannique $»$ en quittant la PCP, car sinon « d'autres vont s'emparer de nos quotas $»^{29}$. Dans la double perspective des élections législatives de mai 1997 et de la signature du traité d'Amsterdam, la composante dite « eurosceptique » du Parti conservateur et le Referendum Party de Jimmy Goldsmith ont également placé la captation de quotas et l'arrêt Factortame en tête de leur argumentaire anti-européen. En transférant le problème dans l'arène électorale, ils ont offert un appui aux organisations de pêcheurs pour obtenir du gouvernement Blair des mesures

\footnotetext{
24. Arrêt Factortame C-221/89 du 13 mars 1991. Voir aussi les arrêts Agegate C-3/87 et Faderow C-216/87.

25. Sur cette idée de contournement de l'État, voir Bertrand Badie et Marie-Claude Smouts, Le Retournement du monde, Paris, Presses de Sciences Po, 2e édition, 1995.

26. Entretien avec Jean-Marie Zarza, Hendaye, 15 juillet 1998.

27. Voir Michel Roux, L'Imaginaire marin des Français. Mythe et géographie de la mer, Paris, L'Harmattan, 1997.

28. Voir Jacques Leruez, Le Royaume-Uni après les élections de mai 1997, Paris, Les Études du CERI n 38, janvier 1998.

29. Austin Mitchell, The Common Fisheries Policies. End or Mend, Londres, Campaign for an Independent Britain, 1996.
} 
nationales établissant, conformément à la jurisprudence de la Cour de Luxembourg, les critères d'un lien économique réel entre tout navire de pêche battant pavillon du Royaume-Uni et « les communautés de pêcheurs ${ }^{30}$ de ce pays. C'est ainsi que,

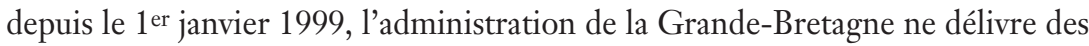
licences de pêche qu'aux navires respectant l'une des quatre conditions suivantes : au moins $50 \%$ des captures d'espèces soumises à quota sont débarquées dans un port britannique ; au moins $50 \%$ de l'équipage réside dans une zone côtière britannique ; un certain nombre de dépenses d'équipement du navire sont effectuées en Grande-Bretagne ; l'armateur est en mesure de présenter d'autres preuves démontrant la réalité du lien économique. À l'avenir, les armements angloespagnols ne pourront donc plus se contenter de mouiller une fois l'an dans leur port d'attache officiel pour la visite technique obligatoire. Ils devront négocier avec l'administration et les communautés portuaires britanniques les termes d'une allégeance imposée.

En France, la captation de quotas n'a jamais été instrumentalisée par les mouvements politiques opposés à l'Union européenne dans une élection nationale. Le lobbying de l'Union des armateurs à la pêche de France et des principaux syndicats de pêcheurs a conduit néanmoins le gouvernement Jospin à faire appliquer, également à compter du $1^{\mathrm{er}}$ janvier 1999, des conditions très proches de celles introduites en Grande-Bretagne ${ }^{31}$.

Ces politiques réactives tant britannique que française démontrent bien que la capacité des États à protéger leur économie nationale et leurs communautés professionnelles (pêcheurs, mais aussi dockers, employés des criées et des chantiers navals) n'a pas été réduite à néant par la disposition des capteurs de quotas à se projeter dans l'espace européen pour y mener une nouvelle forme d'activité transnationale. Ayant perdu le monopole de la fixation des normes, l'État doit néanmoins négocier en permanence avec les capteurs de quotas et s'attendre à tout moment à ce que ces derniers accroissent leur marge de manœuvre en opposant - via le droit de la Communauté européenne - la légitimité du marché européen à celle du territoire national. L'obligation faite aux navires franco-espagnols, en vertu du code français du travail maritime, d'avoir à leur bord un capitaine et un second de nationalité française, au motif que ceux-ci exercent des fonctions relatives à l'ordre et à la sécurité publics - comme la rédaction de l'état-civil - pourrait ainsi être l'objet de prochains contentieux fort intéressants devant les juges européens pour atteinte au principe de la libre circulation des travailleurs ${ }^{32}$. 
$\boldsymbol{L}$ 'opposition entre des communautés portuaires accrochées au primat du territoire et des capteurs de quotas défendant la logique du marché européen tend à s'estomper à mesure que les premières multiplient les échanges transnationaux avec les seconds. Les intérêts qui unissent l'armement franco-espagnol Pronaval au port de La Rochelle en est un bon exemple. En contrepartie d'une criée lui permettant d'écouler des poissons dont la demande est plus forte sur le marché français que sur le marché espagnol, l'armement contribue par ses apports (16 \% du tonnage local en 1998) à assainir la situation du port charentais qui a connu un fort déclin d'activités au cours des années quatre-vingt-dix. Il n'en demeure pas moins que, dans un tel schéma favorisé par l'Europe, les communautés portuaires se trouvent confrontées à une incertitude sociale croissante, les capteurs de quotas étant susceptibles à tout moment de déplacer leurs activités là où les conditions du marché leur sembleraient meilleures.

En outre, la captation de quotas pose ouvertement la question du rapport entre la libéralisation des activités économiques au sein de l'Union européenne et le respect de règles sociales qui constituent un fondement essentiel des États-providence. On a vu que les armements franco-espagnols choisissent d'inscrire la majorité de leurs équipages à la caisse sociale des marins espagnols, dont les cotisations sont moins élevées. Seulement, en l'état actuel, l'administration française n'a guère les moyens de contrôler si les versements s'effectuent régulièrement auprès de l'organisme espagnol compétent ; et l'administration espagnole ne cherche pas davantage à le faire, puisque les armements sont enregistrés en France. La protection des hommes d'équipage s'en trouve fragilisée faute, pour l'instant, d'un rapprochement suffisant des politiques sociales entre États membres.

\footnotetext{
30. Remarquons que les mesures prises par le ministère britannique de l'Agriculture s'intitulent « The licensing of fishing vessels and maintaining of economic links with the fishing communities of the United Kingdom » (souligné par nous).

31. Voir le décret du ministre de l'Agriculture du 31 août 1998 en application de la loi d'orientation sur la pêche n 97-1051 du 18 novembre 1997.

32. Voir Michel Morin, « La condition de nationalité du capitaine de navire français », à paraître en 1999 dans l'Annuaire de Droit Maritime et Océanique.
} 